\title{
PP2A Phosphatase as an Emerging Viral Host Factor
}

\author{
Michal Slawomir Barski, Jordan James Minnell ${ }^{\dagger}$ and Goedele Noella Maertens ${ }^{*}$ \\ Department of Infectious Disease, Section of Molecular Virology, St Mary's Hospital, Imperial College London, \\ London, United Kingdom
}

OPEN ACCESS

Edited by:

Chen Zhao,

Fudan University, China

Reviewed by:

Joaquin Arino,

Universitat Autònoma de Barcelona,

Spain

Manjula Kalia,

Regional Centre for Biotechnology

(RCB), India

*Correspondence:

Goedele Noella Maertens

g.maertens@imperial.ac.uk

${ }^{\dagger}$ Present address:

Jordan James Minnell,

Malaghan Institute of Medical

Research, Victoria

University of Wellington,

Wellington, New Zealand

Specialty section:

This article was submitted to

Virus and Host,

a section of the journal

Frontiers in Cellular and

Infection Microbiology

Received: 16 June 2021

Accepted: 20 July 2021

Published: 04 August 2021

Citation:

Barski MS, Minnell JJ and

Maertens GN (2021) PP2A

Phosphatase as an Emerging

Viral Host Factor.

Front. Cell. Infect. Microbiol. 11:725615.

doi: 10.3389/fcimb.2021.725615
Protein phosphatase 2A (PP2A) is one of the most ubiquitous cellular proteins and is responsible for the vast majority of Ser/Thr phosphatase activity in eukaryotes. PP2A is a heterotrimer, and its assembly, intracellular localization, enzymatic activity, and substrate specificity are subject to dynamic regulation. Each of its subunits can be targeted by viral proteins to hijack and modulate its activity and downstream signaling to the advantage of the virus. Binding to PP2A is known to be essential to the life cycle of many viruses and seems to play a particularly crucial role for oncogenic viruses, which utilize PP2A to transform infected cells through controlling the cell cycle and apoptosis. Here we summarise the latest developments in the field of PP2A viral targeting; in particular recent discoveries of PP2A hijacking through molecular mimicry of a B56-specific motif by several different viruses. We also discuss the potential as well as shortcomings for therapeutic intervention in the face of our current understanding of viral PP2A targeting.

Keywords: PP2A (protein phosphatase 2A), B56-PP2A, LxxIxE SLiM, integrase, viral PP2A targeting

\section{INTRODUCTION}

Reversible protein phosphorylation is a crucial and ubiquitous post-translational modification (PTM). One third of all proteins are thought to experience phosphorylation on the hydroxylated sidechains of serine, threonine or tyrosine residues in an equilibrium maintained by the antagonistic actions of protein kinases and phosphatases. These enzymes are classified based on their substrates as modifying either Ser/Thr or Tyr residues. Although the substrate pool is identical, the number of Ser/Thr phosphatases encoded in the human genome is vastly outnumbered by the kinases (Chen et al., 2017) and is divided into metal-dependent phosphatases (PPM), or phosphoprotein phosphatases (PPP) by virtue of sequence and fold (Chen et al., 2017). There are seven PPP families, the largest of which is the protein phosphatase $2 \mathrm{~A}$ (PP2A), accounting for up to $1 \%$ of the total cellular protein content (Ruediger et al., 1991). PP2A has been coined the "master regulator of the cell cycle" as it regulates every stage of the cell cycle (Wlodarchak and Xing, 2016). It is known to dephosphorylate over 300 cellular substrates (Wlodarchak and Xing, 2016), and is involved in diverse cellular processes including development, cell proliferation and death, cell mobility and cytoskeletal dynamics, the cell cycle and signaling. In addition to its role in neurodevelopmental disorders (Houge et al., 2015; Loveday et al., 2015; Shang et al., 2016; Reynhout et al., 2019; Biswas et al., 2020; Lenaerts et al., 2021), PP2A is mostly known as a tumour suppressor and its inhibition caused by mutation, biochemical downregulation or drug binding is implicated in many cancers (Eichhorn et al., 2009; Ruediger et al., 2011; Ruvolo, 2016; O'Connor et al., 2020). 
It is no surprise that viruses have evolved to target PP2A via several different mechanisms. The aim of this Mini Review is to summarise the different strategies viruses use to hijack PP2A to modulate its phosphatase activity and downstream signaling functions to subsequently enhance viral replication. This is the first review synthesizing our current understanding and the emerging role of LxxIxE motif mimicry-mediated PP2A targeting by viruses.

\section{STRUCTURE, FUNCTION AND REGULATION OF PP2A}

Despite their crucial role in cellular signaling, phosphatases have been, until recently, largely understudied compared to kinases. This is likely due to their complex structure and a multitude of oligomeric forms present in the cell (Shi, 2009). The PP2A phosphatase is a heterotrimer, composed of a horseshoe-like, alpha-helical HEAT repeat scaffold (A) subunit bound to the regulatory (B) and the catalytic $(\mathrm{C})$ subunits. Both $\mathrm{A}$ and $\mathrm{C}$ subunits have two ( $\alpha$ and $\beta$ ) variants, while the $B$ subunit comprises four families (B55/PR55, B56/PR61, PR48/PR72/ PR130 and PR93/striatin). Additionally, each family contains 2-5 isoforms and each one can have alternative splice and translational variants. PP2A exists mainly in two forms, either as the core dimer comprised of the $\mathrm{A}$ and $\mathrm{C}$ subunits, or as the heterotrimer where the core is bound to any one of the B-type regulatory subunits. It is the large number of possible unique structural PP2A permutations which exhibit diversity in both substrate specificity and subcellular localization (Janssens and Goris, 2001). Although the various B subunits recognize a similar region of A (Lenaerts et al., 2021), they lack structural or functional redundancy and are critical regulators for cell survival (Strack et al., 2004; Seshacharyulu et al., 2013).

PP2A regulates the cell cycle by affecting several critical pathways (Wlodarchak and Xing, 2016) including Wnt, mammalian target of rapamycin (mTOR), mitogen activated protein kinase (MAPK) and the phosphoinositide 3-kinase (PI3K). PP2A-B56 is also involved in the regulation of spindle assembly (Espert et al., 2014; Hayward et al., 2019), the cell cycle (Janssens and Goris, 2001), apoptosis (Janssens and Rebollo, 2012), DNA damage response (Peng and Maller, 2010) and chromosome segregation during meiosis (Bel Borja et al., 2020).

The vast range of cellular processes regulated by the different PP2A holoenzymes combined with the modular nature of holoenzyme assembly and lack of redundancy in function presents a perfect node for the deregulation of key cellular processes by tumors and viruses. Indeed, the PP2A genes and their regulators are tumor suppressors that are perturbed at a low (but significant) frequency in human cancers (Sangodkar et al., 2016). PP2A mutations are implicated in lung, breast, colorectal, among other cancers (Wang et al., 1998; Takagi et al., 2000; Ruediger et al., 2001b; Tamaki et al., 2004), where downregulation of PP2A results in transformation - yet minimal activity is essential for cell survival (Ruvolo, 2016). Concurrent with its role as a tumour suppressor, pharmacological inhibitors of PP2A catalytic function such as okadaic acid and microcystin act as potent tumor promoters (MacKintosh and MacKintosh, 1994). Such small molecule inhibitors were initially discovered from screens of natural products and are usually toxins produced by microbes and animals (McCluskey et al., 2002). For example, microcystin and nodularins were purified from blue-green algae, and okadaic acid was firstly identified in the marine sponge Halicondria okadai (Holmes et al., 1990; Dounay and Forsyth, 2002), while tautomycin is produced by Streptomyces verticillatus (MacKintosh and Klumpp, 1990). As such, these could be considered the first exogenous factors identified to affect PP2A activity.

Recent advancements in the structural and biochemical research on PP2A have allowed a much greater understanding of the mechanisms of its exogenous and endogenous regulation as well as effects of carcinogenic mutations (Xu et al., 2006; Cho and $\mathrm{Xu}, 2007$; Xu et al., 2008; Wlodarchak et al., 2013; Jeong et al., 2021). Most PP2A mutations identified in cancer patients are located within PP2A A $\alpha$ and $A \beta$ subunits and affect the binding of other PP2A subunits to the scaffold (Ruediger et al., 2001a; Sablina et al., 2007). Post-translational modifications of the catalytic and scaffold subunits such as phosphorylation and methylation modulate the activity of PP2A in a similar way, also affecting the association with regulatory subunits (Janssens and Goris, 2001; Janssens and Rebollo, 2012; Seshacharyulu et al., 2013). Similarly, phosphorylation of B subunits regulates the sub-cellular localization of the holoenzyme affecting its substrate repertoire (Strack et al., 1998).

\section{PP2A IS UBIQUITOUSLY TARGETED AND MODULATED BY VIRUSES}

Numerous proteins from a wide range of viral families have already been identified to interact with and modulate the activity of PP2A (Figure 1, and extensively reviewed by (Guergnon et al., 2011)). The first known case was the small $\mathrm{T}$ antigen (sT) of the oncogenic simian virus 40 (SV40). sT and large T antigen (LT) of SV40, merkell cell polyomavirus (MCV), or Murine polyoma virus $(\mathrm{Py})$ are alternatively-spliced oncoproteins which play a role in the transformation of infected cells (Rundell and Parakati, 2001; Yu et al., 2001; Kwun et al., 2009). This property of sT, however, is fully reliant on its binding to the scaffold subunit of PP2A (Yang et al., 1991; Ruediger et al., 1992), which displaces the regulatory subunit (Pallas et al., 1990; Chen et al., 2004) and therefore inhibits PP2A-mediated dephosphorylation of many substrates (with the known exception of histone $\mathrm{H} 1$ ) and promotes viral (DNA) replication (Scheidtmann et al., 1991; Sablina et al., 2007; Kwun et al., 2009; Bollag et al., 2010; Bhat et al., 2020). A close dissection of this interaction has been made possible through the crystal structure of the SV40 sT in complex with the A $\alpha$ subunit of PP2A (Cho et al., 2007). Conversely, the West Nile virus capsid (WNVC) protein upregulates PP2Amediated activity hereby inhibiting AP-1 dependent transcription (Hunt et al., 2007). 


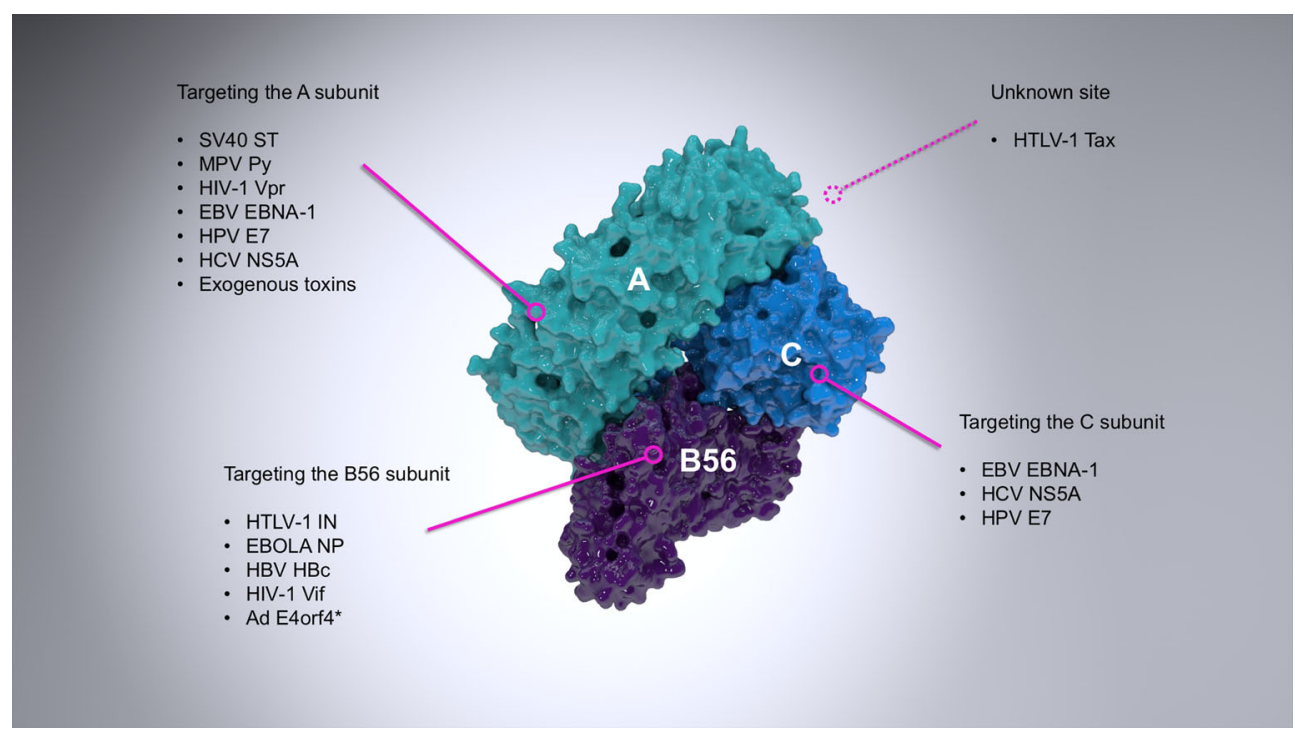

FIGURE 1 | A summary of the most important examples of PP2A targeting by viruses. PP2A is a heterotrimer composed of the scaffold (A; cyan), regulatory (B56; purple) and catalytic (C; light blue) subunits. The figure shows a B56-containing PP2A holoenzyme (PDB ID: 2IAE). Viral proteins interact with PP2A via different subunits, as indicated where it is known. The vast majority of viral proteins known to interact with the regulatory subunit of PP2A target exclusively B56, although the adenovirus E4orf4 (asterisked above) can also target B55 subunits (Kleinberger, 2020) and some other viral proteins are known to associate exclusively with other regulatory subunits (for example the polymerase of rinderpest virus (Sleeman and Baron, 2005).

Several other oncogenic viruses target PP2A in order to inhibit its downstream pro-apoptotic signaling, therefore leading to transformation and immortalization. Examples include the E7 protein of human papillomaviruses (HPV) (Pim et al., 2005) or the leader protein (EBNA-LP) encoded by the Epstein-Barr virus (EBV) (Garibal et al., 2007). At the same time, other viruses target PP2A to drive apoptosis, such as the E4orf4 protein of adenovirus (Kleinberger and Shenk, 1993) and the Py sT-antigen (Bhat et al., 2020).

The innate immune response is the first line of defense upon pathogen invasion. PP2A inhibits this process by dephosphorylating interferon regulatory transcription factor 3 thus blocking expression of interferon stimulated genes (Wang et al., 2020a). Many viruses depend on (Xu et al., 2019), or exploit this process, either by stimulating (Zan et al., 2020), or upregulating PP2A (Duong et al., 2004; Christen et al., 2007) thereby blocking type I IFN response in favor of increasing viral replication.

The two retroviruses of clinical importance: human immunodeficiency virus type $1(\mathrm{HIV}-1)$ and human $\mathrm{T}$ lymphotropic virus type 1 (HTLV-1) have also evolved several independent mechanisms of PP2A hijacking. The Vpr protein of HIV-1 associates with the scaffold subunit of PP2A (Godet et al., 2010) while HIV-1 Vif targets the PP2A regulatory B56 subunit for degradation (Greenwood et al., 2016; Naamati et al., 2019; Marelli et al., 2020; Nagata et al., 2020). Both processes act independently to lead to $\mathrm{G} 2 / \mathrm{M}$ cell cycle arrest. The transactivator protein (Tax) of HTLV-1 is one of the most potent oncogenes known (Mohanty and Harhaj, 2020) and associates with an unidentified region of PP2A as part of a ternary complex with IKK $\gamma$ (Fu et al., 2003; Merling et al., 2007). Inhibition of PP2A activity prevents dephosphorylation of IKK $\gamma$ which leads to activation of IKK $\gamma$-regulated genes and ultimately cell cycle arrest. HTLV-1 integrase (IN) associates with the B56 subunit to enhance infection and might play a role in integration site targeting (Maertens, 2016; Barski et al., 2020; Bhatt et al., 2020). The relevance of cell senescence induced by both HIV-1 and HTLV-1 in the context of infection remains unknown.

It remains to be understood how viral hijacking of PP2A can lead to such vastly different biochemical and phenotypic events. Unfortunately, the structure-driven mechanistic understanding of these processes is still scarce, with the aforementioned sT:A $\alpha$ and the IN:B56r (described below) - the only viral protein complexes resolved with PP2A thus far.

\section{EMERGING ROLE OF VIRAL PP2A TARGETING VIA LXXIXE MOTIF MIMICRY}

An emerging mechanism of PP2A binding employed by viruses relies on mimicking a short linear motif (SLiM) used by endogenous substrates and partners of PP2A to bind to all isoforms of the B56 regulatory subunit (Hertz et al., 2016; Wang et al., 2016). The SLiM of the consensus sequence LxxIxE (where ' $x$ ' can be any amino acid) is located on intrinsically disordered regions of many PP2A endogenous partners and becomes fixed upon binding to a highlyconserved groove between the third and fourth HEAT repeat in the centre of the concave face of the B56 subunit of PP2A. Structural studies focusing on BubR1 and RepoMan have 
identified the interaction interface largely defined by the Leu pocket (B56 residues K183, T184, H187, R188, E226, I227) and Ile pocket (B56 residues H187, Y190, I227, I231) (Wang et al., 2016). Interactions, both hydrophobic and electrostatic in nature, involve every residue of the LxxIxE SLiM and SLiM point mutations weaken the interaction. At the same time, Wang et al. showed that the presence of a phosphorylated serine following the SLiM leucine significantly enhances binding through a salt bridge interaction with R188. The limitation of the aforementioned studies is the use of short peptides mimicking the SLiM region rather than entire domains/subunits.

We recently discovered through proteomic mass spectrometry approaches that PP2A-B56 is a functional binding partner of the deltaretroviral integrase enzyme (Maertens, 2016). IN catalyses the insertion of reversetranscribed viral DNA (vDNA) into host chromatin which is a key step in the deltaretroviral replication cycle. In vitro strandtransfer activity of deltaretroviral IN was largely stimulated in the presence of B56. Mutational scanning identified the B56 region encompassing HEAT repeats 3 and 4 critical to IN binding and strand-transfer stimulation. The long linker between the IN catalytic domain (CCD) and the C-terminal domain (CTD) encompasses the LxxIxE sequence - a bona fide B56-targeting motif. Indeed, every single point mutation within the simian Tcell lymphotropic virus type 1 (STLV-1) IN SLiM (L213A, P214A, P215A/P217A, I216A, E218A) reduced IN binding to $\mathrm{B} 56$ and in vitro strand-transfer activity stimulation in presence of B56 (Barski et al., 2020). Conversely, B56 mutations L194A, R197A located in the LxxIxE-binding groove also prevented this functional interaction (Maertens, 2016).

The X-ray crystallographic structure of deltaretroviral human lymphotropic virus type-1 (HTLV-1) IN residues 200-297, encompassing the LxxIxE SLiM, and B56 revealed a binding interface akin to that of BubR1 and RepoMan (Figures 2A, B), yet devoid of the phosphoserine-R188 interaction (Barski et al., 2020). Our understanding of the IN-PP2A binding was then largely expanded by two independent studies by Barski et al. (2020) and Bhatt et al. (2020) who have respectively reported structures of STLV-1 and HTLV-1 intasomes (IN:vDNA supramolecular intermediates) in complexes with B56. Both structures corroborated observations from the co-crystal complex but also identified a previously-unknown ligand binding site on B56. The LxxIxE SLiM seems to have evolved strategically to exploit the oligomeric structure of the intasome in order to further stabilize the IN:B56 complex by repurposing the LxxIxE SLiM on a neighboring IN subunit (Figure 2C). Effectively, the two SLiMs - contributed by a dimer of IN bind two separate sites on B56. The secondary site runs perpendicular to the primary SLiM binding groove and involves residues E78, T81, H82, N83 and R143 located on the solvent-exposed surface of HEAT repeats five and six.

Although the exact role of PP2A in deltaretroviral integration has not been discerned yet, it is an essential host factor for viral replication - shRNA knockdown of B56 $\gamma$ significantly reduced HTLV-1 infectivity (Bhatt et al., 2020). Even though PP2A does not directly associate with chromatin, it dephosphorylates many transcription factors and chromatin modifiers (Eichhorn et al., 2009; Shanker et al., 2013) including those whose binding sites were found in the vicinity of HTLV-1 integration sites (Melamed et al., 2013). Binding of IN to PP2A does not occlude the active site (Barski et al., 2020; Bhatt et al., 2020) suggesting IN could modulate PP2A catalytic activity to dephosphorylate itself or other targets, although clearly the presence of phosphorylated IN is not essential for binding. PP2A might also play a role in targeting the pre-integration complex to the site of integration, as has been known for IN-associated host factors in other retroviral families (Kvaratskhelia et al., 2014).

Interestingly, Wang et al. recently also identified a binding site on B56 for yet another motif. This "acidic patch" on B56 is located in close proximity to the primary LxxIxE SLiM binding sites utilized by deltaretroviral INs (Wang et al., 2020b). In the structures of STLV/HTLV intasome:B56 complexes the "acidic patch" identified by Wang et al. is not occupied. It is argued that a subset of LxxIxE-containing endogenous partners and substrates of PP2A need an additional site as the SLiM binding to the primary site on B56 only confers micromolar-range affinities. It is tempting to speculate that the divergence in mechanisms of binding between endogenous and deltaretroviral PP2A binders (that have been demonstrated so far) could be exploited for antiviral drug design to avoid side effects caused by inhibition of PP2A dephosphorylation activity in key cellular pathways. Such an approach may also mean that the same inhibitor could be used against more than one virus. Additionally, targeting a site on a host protein, rather than a viral protein minimizes the problem of drug resistance.

Although mostly known for its ability to target APOBEC3 restriction factors for proteasomal degradation (Desimmie et al., 2014; Harris and Dudley, 2015), intense studies on the HIV-1 Vif accessory protein revealed that the cell cycle arrest induced by Vif occurs through its targeted degradation of B56 (Greenwood et al., 2016; Marelli et al., 2020; Nagata et al., 2020; Salamango and Harris, 2020). Interestingly, although Vif does not encode an LxxIxE SLiM motif, co-expression of an LxxIxE-like peptide inhibitor known to outcompete B56 binders reduced degradation of B56 in a dose-dependent manner (Salamango et al., 2020) suggesting that Vif recognition by B56 involves residues surrounding the LxxIxE SLiM binding pocket.

The panel of LxxIxE SLiM-utilising viruses includes filoviruses (Hertz et al., 2016) and it has been shown that the most clinically-important filovirus, the Ebola virus (EBOV), associates with B56 via the SLiM located on its nucleoprotein (NP). Although structural details of this interaction are still missing, Kruse et al. determined that NP associates with PP2A-B56 to dephosphorylate VP30, an EBOV-encoded protein and binding partner of NP (Kruse et al., 2018). VP30 is an EBOV-specific transcription factor, regulated by phosphorylation (Takamatsu et al., 2020), and essential for initiation of viral transcription (Tigabu et al., 2018). The NP LxxIxE, and hence its interaction with B56, was found to be required for EBOV transcription, but not replication. Interestingly, phosphorylation of VP30 increases its affinity for NP (Biedenkopf et al., 2013) which in turn increases its chances 

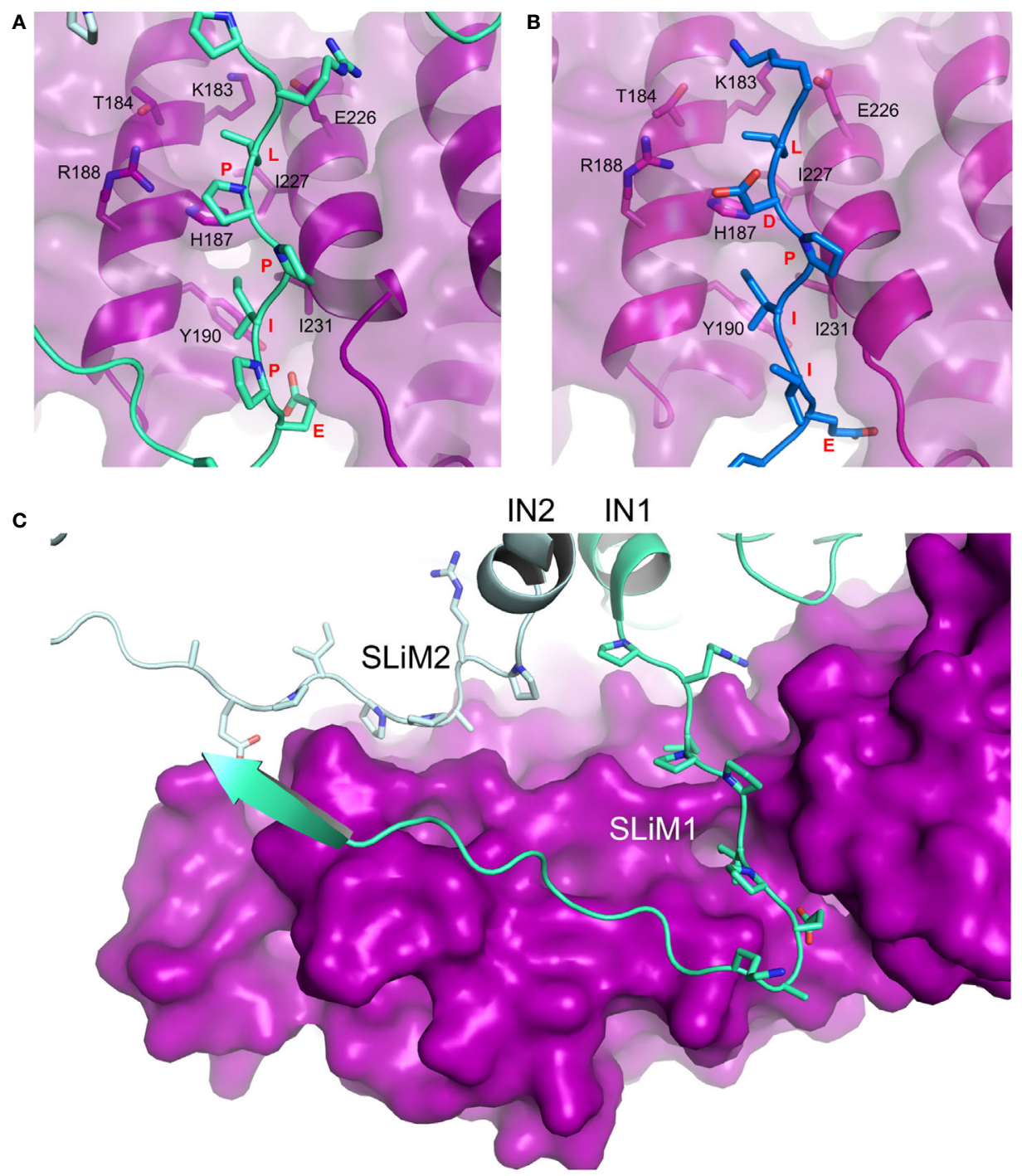

FIGURE 2 | Hijacking of PP2A via the B56-binding LxxIxE SLiM. (A) Details of LPPIPE IN SLiM (equivalent to SLiM1 in panel C; green) binding to the canonical LxxlxE-binding groove on B56 (purple). Interacting residues are shown as sticks (PDB ID: 6Z2Y). (B) BUBR1 LDPIIE SLiM (also equivalent to SLiM1 in panel C; blue) binding to the same site on B56 (purple) (PDB ID: 5JJA). In this case, the aspartate was used as a phosphomimetic. The conformational overlap between residues of the SLiMs and the binding groove in both structures is evident. (C) Deltaretroviral integrase (IN) dimer contributes two identical SLiM regions to bind one molecule of B56 (purple) at two different sites. Each IN chain (IN1 and IN2) is coloured with a different shade of green (PDB ID: 6Z2Y, EMDB: 11052). PyMOL software was used for analysis.

of PP2A-mediated dephosphorylation - likely forming a negative feedback loop to balance EBOV transcription and replication.

Another example of viral B56 recruitment via the LxxIxE SLiM was recently discovered in the hepatitis B virus (HBV) capsid or core protein ( $\mathrm{HBc}$ ) (Xi et al., 2021). $\mathrm{HBc}$ is a multifunctional protein taking part in every step of $\mathrm{HBV}$ replication: from its role as the nucleocapsid to reverse transcription, to vDNA nuclear import and packaging (Nassal, 1992; Wynne et al., 1999; Liu et al., 2015). HBc is regulated through phosphorylation of its CTD - supporting different steps of the HBV viral life cycle depending on the level of CTD phosphorylation (Ludgate et al., 2016; Luo et al., 2020). The
SLiM is located on the NTD-CTD linker whose truncation was previously reported to affect multiple aspects of $\mathrm{HBV}$ replication as well as NTD assembly and the phosphorylation of the CTD (Liu et al., 2018). Point mutations within the HBc LxxIxE caused multiple pleiotropic effects in the HBV life cycle resulting in reduced HBV infectivity. Surprisingly, the HBc SLiM slightly diverges from the canonical LxxIxE consensus sequence, encoding a leucine instead of the isoleucine (LSTLPE). Although no direct interaction between $\mathrm{HBc}$ and B56 was identified, it remains possible that the interaction is very transient in nature and/or relies on varying degrees of $\mathrm{HBc}$ phosphorylation. 


\section{CONCLUSIONS}

PP2A is a promising anti-cancer drug target (O'Connor et al., 2018; Leonard et al., 2020) and PP2A targeted agents are currently used in combination therapy in cancer (Mazhar et al., 2019). Here, we have synthesized efforts of the last few years in broadening our understanding of viral PP2A targeting; particularly via the LxxIxE-mediated B56 binding. Utilizing PP2A's vast repertoire of functions and partners seems to be crucial for many clinically-relevant viruses such as Ebola, West Nile virus, HIV-1, HTLV-1 and many oncogenic viruses. It is imperative to further our understanding of PP2A hijacking and once more structural and mechanistic details of this phenomenon are obtained, PP2A might present an opportunity for antiviral pharmacological interventions.

\section{REFERENCES}

Barski, M. S., Minnell, J. J., Hodakova, Z., Pye, V. E., Nans, A., Cherepanov, P., et al. (2020). Cryo-EM Structure of the Deltaretroviral Intasome in Complex With the PP2A Regulatory Subunit B56gamma. Nat. Commun. 11, 5043. doi: 10.1038/s41467-020-18874-y

Bel Borja, L., Soubigou, F., Taylor, S. J. P., Fraguas Bringas, C., Budrewicz, J., LaraGonzalez, P., et al. (2020). BUB-1 Targets PP2A:B56 to Regulate Chromosome Congression During Meiosis I in C. Elegans Oocytes. Elife 9. doi: 10.7554/ eLife.65307

Bhat, S. A., Sarwar, Z., Gillani, S. Q., Un Nisa, M., Reshi, I., Nabi, N., et al. (2020). Polyomavirus Small $\mathrm{T}$ Antigen Induces Apoptosis in Mammalian Cells Through the UNC5B Pathway in a PP2A-Dependent Manner. J. Virol. 94. doi: $10.1128 /$ JVI.02187-19

Bhatt, V., Shi, K., Salamango, D. J., Moeller, N. H., Pandey, K. K., Bera, S., et al. (2020). Structural Basis of Host Protein Hijacking in Human T-Cell Leukemia Virus Integration. Nat. Commun. 11, 3121. doi: 10.1038/s41467-020-16963-6

Biedenkopf, N., Hartlieb, B., Hoenen, T., and Becker, S. (2013). Phosphorylation of Ebola Virus VP30 Influences the Composition of the Viral Nucleocapsid Complex: Impact on Viral Transcription and Replication. J. Biol. Chem. 288, 11165-11174. doi: 10.1074/jbc.M113.461285

Biswas, D., Cary, W., and Nolta, J. A. (2020). PPP2R5D-Related Intellectual Disability and Neurodevelopmental Delay: A Review of the Current Understanding of the Genetics and Biochemical Basis of the Disorder. Int. J. Mol. Sci. 21. doi: 10.3390/ijms 21041286

Bollag, B., Hofstetter, C. A., Reviriego-Mendoza, M. M., and Frisque, R. J. (2010). JC Virus Small T Antigen Binds Phosphatase PP2A and Rb Family Proteins and Is Required for Efficient Viral DNA Replication Activity. PloS One 5, e10606. doi: 10.1371/journal.pone.0010606

Chen, M. J., Dixon, J. E., and Manning, G. (2017). Genomics and Evolution of Protein Phosphatases. Sci. Signal. 10. doi: 10.1126/scisignal.aag1796

Chen, W., Possemato, R., Campbell, K. T., Plattner, C. A., Pallas, D. C., and Hahn, W. C. (2004). Identification of Specific PP2A Complexes Involved in Human Cell Transformation. Cancer Cell 5, 127-136. doi: 10.1016/S1535-6108(04) 00026-1

Cho, U. S., Morrone, S., Sablina, A. A., Arroyo, J. D., Hahn, W. C., and Xu, W. (2007). Structural Basis of PP2A Inhibition by Small T Antigen. PloS Biol. 5, e202. doi: 10.1371/journal.pbio.0050202

Cho, U. S., and Xu, W. (2007). Crystal Structure of a Protein Phosphatase 2A Heterotrimeric Holoenzyme. Nature 445, 53-57. doi: 10.1038/nature05351

Christen, V., Treves, S., Duong, F. H. T., and Heim, M. H. (2007). Activation of Endoplasmic Reticulum Stress Response by Hepatitis Viruses Up-Regulates Protein Phosphatase 2A. Hepatology 46, 558-565. doi: 10.1002/hep.21611

Desimmie, B. A., Delviks-Frankenberrry, K. A., Burdick, R. C., Qi, D., Izumi, T., and Pathak, V. K. (2014). Multiple APOBEC3 Restriction Factors for HIV-1 and One Vif to Rule Them All. J. Mol. Biol. 426, 1220-1245. doi: 10.1016/ j.jmb.2013.10.033

\section{AUTHOR CONTRIBUTIONS}

$\mathrm{MB}, \mathrm{JM}$ and GM wrote the Review. All authors contributed to the article and approved the submitted version.

\section{FUNDING}

$\mathrm{MB}$, JM and GM are funded by the Wellcome Trust ([Investigator Award 107005 to GM]).

\section{ACKNOWLEDGMENTS}

We apologize to colleagues whose work we were unable to cite due to space constraints.

Dounay, A. B., and Forsyth, C. J. (2002). Okadaic Acid: The Archetypal Serine/ Threonine Protein Phosphatase Inhibitor. Curr. Med. Chem. 9, 1939-1980. doi: $10.2174 / 0929867023368791$

Duong, F. H. T., Filipowicz, M, Tripodi, M., La Monica, N., and Heim, M. H. (2004). Hepatitis C Virus Inhibits Interferon Signaling Through UpRegulation of Protein Phosphatase 2A. Gastroenterology 126, 263-277. doi: 10.1053/j.gastro.2003.10.076

Eichhorn, P. J., Creyghton, M. P., and Bernards, R. (2009). Protein Phosphatase 2A Regulatory Subunits and Cancer. Biochim. Biophys. Acta 1795, 1-15. doi: 10.1016/j.bbcan.2008.05.005

Espert, A., Uluocak, P., Bastos, R. N., Mangat, D., Graab, P., and Gruneberg, U. (2014). PP2A-B56 Opposes Mps1 Phosphorylation of Knll and Thereby Promotes Spindle Assembly Checkpoint Silencing. J. Cell Biol. 206, 833-842. doi: $10.1083 /$ jcb.201406109

Fu, D. X., Kuo, Y. L., Liu, B. Y., Jeang, K. T., and Giam, C. Z. (2003). Human TLymphotropic Virus Type I Tax Activates I-Kappa B Kinase by Inhibiting IKappa B Kinase-Associated Serine/Threonine Protein Phosphatase 2A. J. Biol. Chem. 278, 1487-1493. doi: 10.1074/jbc.M210631200

Garibal, J., Hollville, E., Bell, A. I., Kelly, G. L., Renouf, B., Kawaguchi, Y., et al. (2007). Truncated Form of the Epstein-Barr Virus Protein EBNA-LP Protects Against Caspase-Dependent Apoptosis by Inhibiting Protein Phosphatase 2A. J. Virol. 81, 7598-7607. doi: 10.1128/JVI.02435-06

Godet, A. N., Guergnon, J., Croset, A., Cayla, X., Falanga, P. B., Colle, J. H., et al. (2010). PP2A1 Binding, Cell Transducing and Apoptotic Properties of Vpr(7792): A New Functional Domain of HIV-1 Vpr Proteins. PloS One 5, el3760. doi: 10.1371/journal.pone. 0013760

Greenwood, E. J., Matheson, N. J., Wals, K., van den Boomen, D. J., Antrobus, R, Williamson, J. C., et al. (2016). Temporal Proteomic Analysis of HIV Infection Reveals Remodelling of the Host Phosphoproteome by Lentiviral Vif Variants. Elife 5. doi: 10.7554/eLife.18296

Guergnon, J., Godet, A. N., Galioot, A., Falanga, P. B., Colle, J. H., Cayla, X., et al. (2011). PP2A Targeting by Viral Proteins: A Widespread Biological Strategy From DNA/RNA Tumor Viruses to HIV-1. Biochim. Biophys. Acta 1812, 1498-1507. doi: 10.1016/j.bbadis.2011.07.001

Harris, R. S., and Dudley, J. P. (2015). APOBECs and Virus Restriction. Virology 479-480, 131-145. doi: 10.1016/.j.virol.2015.03.012

Hayward, D., Bancroft, J., Mangat, D., Alfonso-Perez, T., Dugdale, S., McCarthy, J., et al. (2019). Checkpoint Signaling and Error Correction Require Regulation of the MPS1 T-Loop by PP2A-B56. J. Cell Biol. 218, 3188-3199. doi: 10.1083/ jcb. 201905026

Hertz, E. P. T., Kruse, T., Davey, N. E., Lopez-Mendez, B., Sigurethsson, J. O. Montoya, G., et al. (2016). A Conserved Motif Provides Binding Specificity to the PP2A-B56 Phosphatase. Mol. Cell 63, 686-695. doi: 10.1016/ j.molcel.2016.06.024

Holmes, C. F., Luu, H. A., Carrier, F., and Schmitz, F. J. (1990). Inhibition of Protein Phosphatases-1 and -2A With Acanthifolicin. Comparison With Diarrhetic Shellfish Toxins and Identification of a Region on Okadaic Acid 
Important for Phosphatase Inhibition. FEBS Lett. 270, 216-218. doi: 10.1016/ 0014-5793(90)81271-O

Houge, G., Haesen, D., Vissers, L. E., Mehta, S., Parker, M. J., Wright, M., et al. (2015). B56delta-Related Protein Phosphatase 2A Dysfunction Identified in Patients With Intellectual Disability. J. Clin. Invest. 125, 3051-3062. doi: 10.1172/JCI79860

Hunt, T. A., Urbanowski, M. D., Kakani, K., Law, L. M., Brinton, M. A., and Hobman, T. C. (2007). Interactions Between the West Nile Virus Capsid Protein and the Host Cell-Encoded Phosphatase Inhibitor, I2PP2A. Cell Microbiol. 9, 2756-2766. doi: 10.1111/j.1462-5822.2007.01046.x

Janssens, V., and Goris, J. (2001). Protein Phosphatase 2A: A Highly Regulated Family of Serine/Threonine Phosphatases Implicated in Cell Growth and Signalling. Biochem. J. 353, 417-439. doi: 10.1042/bj3530417

Janssens, V., and Rebollo, A. (2012). The Role and Therapeutic Potential of Ser/ Thr Phosphatase PP2A in Apoptotic Signalling Networks in Human Cancer Cells. Curr. Mol. Med. 12, 268-287. doi: 10.2174/156652412799218930

Jeong, B. C., Bae, S. J., Ni, L., Zhang, X., Bai, X. C., and Luo, X. (2021). Cryo-EM Structure of the Hippo Signaling Integrator Human STRIPAK. Nat. Struct. Mol. Biol. 28, 290-299. doi: 10.1038/s41594-021-00564-y

Kleinberger, T. (2020). Biology of the Adenovirus E4orf4 Protein: From Virus Infection to Cancer Cell Death. FEBS Lett. 594, 1891-1917. doi: 10.1002/18733468.13704

Kleinberger, T., and Shenk, T. (1993). Adenovirus E4orf4 Protein Binds to Protein Phosphatase 2A, and the Complex Down Regulates E1A-Enhanced junB Transcription. J. Virol. 67, 7556-7560. doi: 10.1128/jvi.67.12.75567560.1993

Kruse, T., Biedenkopf, N., Hertz, E. P. T., Dietzel, E., Stalmann, G., Lopez-Mendez, B., et al. (2018). The Ebola Virus Nucleoprotein Recruits the Host PP2A-B56 Phosphatase to Activate Transcriptional Support Activity of VP30. Mol. Cell 69, 136-145 e136. doi: 10.1016/j.molcel.2017.11.034

Kvaratskhelia, M., Sharma, A., Larue, R. C., Serrao, E., and Engelman, A. (2014). Molecular Mechanisms of Retroviral Integration Site Selection. Nucleic Acids Res. 42, 10209-10225. doi: 10.1093/nar/gku769

Kwun, H. J., Guastafierro, A., Shuda, M., Meinke, G., Bohm, A., Moore, P. S., et al. (2009). The Minimum Replication Origin of Merkel Cell Polyomavirus Has a Unique Large T-Antigen Loading Architecture and Requires Small T-Antigen Expression for Optimal Replication. J. Virol. 83, 12118-12128. doi: 10.1128/ JVI.01336-09

Lenaerts, L., Reynhout, S., Verbinnen, I., Laumonnier, F., Toutain, A., BonnetBrilhault, F., et al. (2021). The Broad Phenotypic Spectrum of PPP2R1ARelated Neurodevelopmental Disorders Correlates With the Degree of Biochemical Dysfunction. Genet. Med. 23, 352-362. doi: 10.1038/s41436020-00981-2

Leonard, D., Huang, W., Izadmehr, S., O'Connor, C. M., Wiredja, D. D., Wang, Z., et al. (2020). Selective PP2A Enhancement Through Biased Heterotrimer Stabilization. Cell 181, 688-701.e616. doi: 10.1016/j.cell.2020.03.038

Liu, K., Luckenbaugh, L., Ning, X., Xi, J., and Hu, J. (2018). Multiple Roles of Core Protein Linker in Hepatitis B Virus Replication. PloS Pathog. 14, e1007085. doi: 10.1371/journal.ppat.1007085

Liu, K., Ludgate, L., Yuan, Z., and Hu, J. (2015). Regulation of Multiple Stages of Hepadnavirus Replication by the Carboxyl-Terminal Domain of Viral Core Protein in Trans. J. Virol. 89, 2918-2930. doi: 10.1128/JVI.03116-14

Loveday, C., Tatton-Brown, K., Clarke, M., Westwood, I., Renwick, A., Ramsay, E., et al. (2015). Mutations in the PP2A Regulatory Subunit B Family Genes PPP2R5B, PPP2R5C and PPP2R5D Cause Human Overgrowth. Hum. Mol. Genet. 24, 4775-4779. doi: 10.1093/hmg/ddv182

Ludgate, L., Liu, K., Luckenbaugh, L., Streck, N., Eng, S., Voitenleitner, C., et al. (2016). Cell-Free Hepatitis B Virus Capsid Assembly Dependent on the Core Protein C-Terminal Domain and Regulated by Phosphorylation. J. Virol. 90, 5830-5844. doi: 10.1128/JVI.00394-16

Luo, J., Xi, J., Gao, L., and Hu, J. (2020). Role of Hepatitis B Virus Capsid Phosphorylation in Nucleocapsid Disassembly and Covalently Closed Circular DNA Formation. PloS Pathog. 16, e1008459. doi: 10.1371/ journal.ppat.1008459

MacKintosh, C., and Klumpp, S. (1990). Tautomycin From the Bacterium Streptomyces Verticillatus. Another Potent and Specific Inhibitor of Protein Phosphatases 1 and 2A. FEBS Lett. 277, 137-140. doi: 10.1016/0014-5793(90) 80828-7
MacKintosh, C., and MacKintosh, R. W. (1994). Inhibitors of Protein Kinases and Phosphatases. Trends Biochem. Sci. 19, 444-448. doi: 10.1016/0968-0004(94) 90127-9

Maertens, G. N. (2016). B'-Protein Phosphatase 2A Is a Functional Binding Partner of Delta-Retroviral Integrase. Nucleic Acids Res. 44, 364-376. doi: 10.1093/nar/gkv1347

Marelli, S., Williamson, J. C., Protasio, A. V., Naamati, A., Greenwood, E. J., Deane, J. E., et al. (2020). Antagonism of PP2A is an Independent and Conserved Function of HIV-1 Vif and Causes Cell Cycle Arrest. Elife 9. doi: 10.7554/eLife. 53036

Mazhar, S., Taylor, S. E., Sangodkar, J., and Narla, G. (2019). Targeting PP2A in Cancer: Combination Therapies. Biochim. Biophys. Acta Mol. Cell Res. 1866, 51-63. doi: 10.1016/j.bbamcr.2018.08.020

McCluskey, A., Sim, A. T., and Sakoff, J. A. (2002). Serine-Threonine Protein Phosphatase Inhibitors: Development of Potential Therapeutic Strategies. J. Med. Chem. 45, 1151-1175. doi: 10.1021/jm010066k

Melamed, A., Laydon, D. J., Gillet, N. A., Tanaka, Y., Taylor, G. P., and Bangham, C. R. (2013). Genome-Wide Determinants of Proviral Targeting, Clonal Abundance and Expression in Natural HTLV-1 Infection. PloS Pathog. 9, e1003271. doi: 10.1371/journal.ppat.1003271

Merling, R., Chen, C., Hong, S., Zhang, L., Liu, M., Kuo, Y. L., et al. (2007). HTLV-1 Tax Mutants That do Not Induce G1 Arrest Are Disabled in Activating the Anaphase Promoting Complex. Retrovirology 4, 35. doi: 10.1186/1742-4690-4-35

Mohanty, S., and Harhaj, E. W. (2020). Mechanisms of Oncogenesis by HTLV-1 Tax. Pathogens 9. doi: 10.3390/pathogens 9070543

Naamati, A., Williamson, J. C., Greenwood, E. J., Marelli, S., Lehner, P. J., and Matheson, N. J. (2019). Functional Proteomic Atlas of HIV Infection in Primary Human CD4+ T Cells. Elife 8. doi: 10.7554/eLife.41431

Nagata, K., Shindo, K., Matsui, Y., Shirakawa, K., and Takaori-Kondo, A. (2020). Critical Role of PP2A-B56 Family Protein Degradation in HIV-1 Vif Mediated G2 Cell Cycle Arrest. Biochem. Biophys. Res. Commun. 527, 257-263. doi: 10.1016/j.bbrc.2020.04.123

Nassal, M. (1992). The Arginine-Rich Domain of the Hepatitis B Virus Core Protein Is Required for Pregenome Encapsidation and Productive Viral Positive-Strand DNA Synthesis But Not for Virus Assembly. J. Virol. 66, 4107-4116. doi: 10.1128/jvi.66.7.4107-4116.1992

O'Connor, C. M., Leonard, D., Wiredja, D., Avelar, R. A., Wang, Z., Schlatzer, D., et al. (2020). Inactivation of PP2A by a Recurrent Mutation Drives Resistance to MEK Inhibitors. Oncogene 39, 703-717. doi: 10.1038/s41388-019-1012-2

O'Connor, C. M., Perl, A., Leonard, D., Sangodkar, J., and Narla, G. (2018). Therapeutic Targeting of PP2A. Int. J. Biochem. Cell Biol. 96, 182-193. doi: 10.1016/j.biocel.2017.10.008

Pallas, D. C., Shahrik, L. K., Martin, B. L., Jaspers, S., Miller, T. B., Brautigan, D. L., et al. (1990). Polyoma Small and Middle T Antigens and SV40 Small T Antigen Form Stable Complexes With Protein Phosphatase 2A. Cell 60, 167-176. doi: 10.1016/0092-8674(90)90726-U

Peng, A., and Maller, J. L. (2010). Serine/Threonine Phosphatases in the DNA Damage Response and Cancer. Oncogene 29, 5977-5988. doi: 10.1038/ onc.2010.371

Pim, D., Massimi, P., Dilworth, S. M., and Banks, L. (2005). Activation of the Protein Kinase B Pathway by the HPV-16 E7 Oncoprotein Occurs Through a Mechanism Involving Interaction With PP2A. Oncogene 24, 7830-7838. doi: 10.1038/sj.onc. 1208935

Reynhout, S., Jansen, S., Haesen, D., van Belle, S., de Munnik, S. A., Bongers, E., et al. (2019). De Novo Mutations Affecting the Catalytic Calpha Subunit of PP2A, PPP2CA, Cause Syndromic Intellectual Disability Resembling Other PP2A-Related Neurodevelopmental Disorders. Am. J. Hum. Genet. 104, 139156. doi: 10.1016/j.ajhg.2018.12.002

Ruediger, R., Pham, H. T., and Walter, G. (2001a). Alterations in Protein Phosphatase 2A Subunit Interaction in Human Carcinomas of the Lung and Colon With Mutations in the A Beta Subunit Gene. Oncogene 20, 1892-1899. doi: 10.1038/sj.onc.1204279

Ruediger, R., Pham, H. T., and Walter, G. (2001b). Disruption of Protein Phosphatase 2A Subunit Interaction in Human Cancers With Mutations in the A Alpha Subunit Gene. Oncogene 20, 10-15. doi: 10.1038/sj.onc.1204059

Ruediger, R., Roeckel, D., Fait, J., Bergqvist, A., Magnusson, G., and Walter, G. (1992). Identification of Binding Sites on the Regulatory A Subunit of Protein Phosphatase 2A for the Catalytic C Subunit and for Tumor Antigens of Simian 
Virus 40 and Polyomavirus. Mol. Cell Biol. 12, 4872-4882. doi: 10.1128/ MCB.12.11.4872

Ruediger, R., Ruiz, J., and Walter, G. (2011). Human Cancer-Associated Mutations in the Aalpha Subunit of Protein Phosphatase 2A Increase Lung Cancer Incidence in Aalpha Knock-in and Knockout Mice. Mol. Cell Biol. 31, 38323844. doi: 10.1128/MCB.05744-11

Ruediger, R., Van Wart Hood, J. E., Mumby, M., and Walter, G. (1991). Constant Expression and Activity of Protein Phosphatase 2A in Synchronized Cells. Mol. Cell Biol. 11, 4282-4285. doi: 10.1128/MCB.11.8.4282

Rundell, K., and Parakati, R. (2001). The Role of the SV40 ST Antigen in Cell Growth Promotion and Transformation. Semin. Cancer Biol. 11, 5-13. doi: 10.1006/scbi.2000.0341

Ruvolo, P. P. (2016). The Broken “Off” Switch in Cancer Signaling: PP2A as a Regulator of Tumorigenesis, Drug Resistance, and Immune Surveillance. BBA Clin. 6, 87-99. doi: 10.1016/j.bbacli.2016.08.002

Sablina, A. A., Chen, W., Arroyo, J. D., Corral, L., Hector, M., Bulmer, S. E., et al. (2007). The Tumor Suppressor PP2A Abeta Regulates the RalA GTPase. Cell 129, 969-982. doi: 10.1016/j.cell.2007.03.047

Salamango, D. J., and Harris, R. S. (2020). Dual Functionality of HIV-1 Vif in APOBEC3 Counteraction and Cell Cycle Arrest. Front. Microbiol. 11, 622012. doi: $10.3389 /$ fmicb. 2020.622012

Salamango, D. J., McCann, J. L., Demir, O., Becker, J. T., Wang, J., Lingappa, J. R., et al. (2020). Functional and Structural Insights Into a Vif/PPP2R5 Complex Elucidated Using Patient HIV-1 Isolates and Computational Modeling. J. Virol. 94. doi: 10.1128/JVI.00631-20

Sangodkar, J., Farrington, C. C., McClinch, K., Galsky, M. D., Kastrinsky, D. B., and Narla, G. (2016). All Roads Lead to PP2A: Exploiting the Therapeutic Potential of This Phosphatase. FEBS J. 283, 1004-1024. doi: 10.1111/febs.13573

Scheidtmann, K. H., Mumby, M. C., Rundell, K., and Walter, G. (1991). Dephosphorylation of Simian Virus 40 Large-T Antigen and P53 Protein by Protein Phosphatase 2A: Inhibition by Small-T Antigen. Mol. Cell Biol. 11, 1996-2003. doi: 10.1128/MCB.11.4.1996

Seshacharyulu, P., Pandey, P., Datta, K., and Batra, S. K. (2013). Phosphatase: PP2A Structural Importance, Regulation and its Aberrant Expression in Cancer. Cancer Lett. 335, 9-18. doi: 10.1016/j.canlet.2013.02.036

Shang, L., Henderson, L. B., Cho, M. T., Petrey, D. S., Fong, C. T., Haude, K. M., et al. (2016). De Novo Missense Variants in PPP2R5D Are Associated With Intellectual Disability, Macrocephaly, Hypotonia, and Autism. Neurogenetics 17, 43-49. doi: 10.1007/s10048-015-0466-9

Shanker, V., Trincucci, G., Heim, H. M., and Duong, H. T. (2013). Protein Phosphatase 2A Impairs IFNalpha-Induced Antiviral Activity Against the Hepatitis C Virus Through the Inhibition of STAT1 Tyrosine Phosphorylation. J. Viral Hepat. 20, 612-621. doi: 10.1111/jvh.12083

Shi, Y. (2009). Serine/Threonine Phosphatases: Mechanism Through Structure. Cell 139, 468-484. doi: 10.1016/j.cell.2009.10.006

Sleeman, K., and Baron, M. D. (2005). The Polymerase (L) Protein of Rinderpest Virus Interacts With the Host Cell Protein Striatin. Virology 332, 225-234. doi: 10.1016/j.virol.2004.11.020

Strack, S., Cribbs, J. T., and Gomez, L. (2004). Critical Role for Protein Phosphatase 2A Heterotrimers in Mammalian Cell Survival. J. Biol. Chem. 279, 47732-47739. doi: 10.1074/jbc.M408015200

Strack, S., Zaucha, J. A., Ebner, F. F., Colbran, R. J., and Wadzinski, B. E. (1998). Brain Protein Phosphatase 2A: Developmental Regulation and Distinct Cellular and Subcellular Localization by B Subunits. J. Comp. Neurol. 392, 515-527. doi: 10.1002/(SICI) 1096-9861(19980323)392:4<515::AID-CNE8>3.0.CO;2-3

Takagi, Y., Futamura, M., Yamaguchi, K., Aoki, S., Takahashi, T., and Saji, S. (2000). Alterations of the PPP2R1B Gene Located at 11q23 in Human Colorectal Cancers. Gut 47, 268-271. doi: 10.1136/gut.47.2.268

Takamatsu, Y., Krahling, V., Kolesnikova, L., Halwe, S., Lier, C., Baumeister, S., et al. (2020). Serine-Arginine Protein Kinase 1 Regulates Ebola Virus Transcription. mBio 11. doi: 10.1128/mBio.02565-19

Tamaki, M., Goi, T., Hirono, Y., Katayama, K., and Yamaguchi, A. (2004). PPP2R1B Gene Alterations Inhibit Interaction of PP2A-Abeta and PP2A-C Proteins in Colorectal Cancers. Oncol. Rep. 11, 655-659. doi: 10.7554/ eLife.41431
Tigabu, B., Ramanathan, P., Ivanov, A., Lin, X., Ilinykh, P. A., Parry, C. S., et al. (2018). Phosphorylated VP30 of Marburg Virus Is a Repressor of Transcription. J. Virol. 92. doi: 10.1128/JVI.00426-18

Wang, X., Bajaj, R., Bollen, M., Peti, W., and Page, R. (2016). Expanding the PP2A Interactome by Defining a B56-Specific SLiM. Structure 24, 2174-2181. doi: 10.1016/j.str.2016.09.010

Wang, S. S., Esplin, E. D., Li, J. L., Huang, L., Gazdar, A., Minna, J., et al. (1998). Alterations of the PPP2R1B Gene in Human Lung and Colon Cancer. Science 282, 284-287. doi: 10.1126/science.282.5387.284

Wang, X., Garvanska, D. H., Nasa, I., Ueki, Y., Zhang, G., Kettenbach, A. N., et al. (2020b). A Dynamic Charge-Charge Interaction Modulates PP2A:B56 Substrate Recruitment. Elife 9. doi: 10.7554/eLife.55966

Wang, J., Li, H., Xue, B., Deng, R., Huang, X., Xu, Y., et al. (2020a). IRF1 Promotes the Innate Immune Response to Viral Infection by Enhancing the Activation of IRF3. J. Virol. 94. doi: 10.1128/JVI.01231-20

Wlodarchak, N., Guo, F., Satyshur, K. A., Jiang, L., Jeffrey, P. D., Sun, T., et al. (2013). Structure of the Ca2+-Dependent PP2A Heterotrimer and Insights Into Cdc6 Dephosphorylation. Cell Res. 23, 931-946. doi: 10.1038/cr.2013.77

Wlodarchak, N., and Xing, Y. (2016). PP2A as a Master Regulator of the Cell Cycle. Crit. Rev. Biochem. Mol. Biol. 51, 162-184. doi: 10.3109/ 10409238.2016.1143913

Wynne, S. A., Crowther, R. A., and Leslie, A. G. (1999). The Crystal Structure of the Human Hepatitis B Virus Capsid. Mol. Cell 3, 771-780. doi: 10.1016/ S1097-2765(01)80009-5

Xi, J., Luckenbaugh, L., and Hu, J. (2021). Multiple Roles of PP2A Binding Motif in Hepatitis B Virus Core Linker and PP2A in Regulating Core Phosphorylation State and Viral Replication. PloS Pathog. 17, e1009230. doi: 10.1371/ journal.ppat.1009230

Xu, Y., Chen, Y., Zhang, P., Jeffrey, P. D., and Shi, Y. (2008). Structure of a Protein Phosphatase 2A Holoenzyme: Insights Into B55-Mediated Tau Dephosphorylation. Mol. Cell 31, 873-885. doi: 10.1016/j.molcel.2008.08.006

Xu, Y., Xing, Y., Chen, Y., Chao, Y., Lin, Z., Fan, E., et al. (2006). Structure of the Protein Phosphatase 2A Holoenzyme. Cell 127, 1239-1251. doi: 10.1016/ j.cell.2006.11.033

Xu, J., Zhang, L., Xu, Y., Zhang, H., Gao, J., Wang, Q., et al. (2019). PP2A Facilitates Porcine Reproductive and Respiratory Syndrome Virus Replication by Deactivating Irf3 and Limiting Type I Interferon Production. Viruses 11. doi: $10.3390 / \mathrm{v} 11100948$

Yang, S. I., Lickteig, R. L., Estes, R., Rundell, K., Walter, G., and Mumby, M. C. (1991). Control of Protein Phosphatase 2A by Simian Virus 40 Small-T Antigen. Mol. Cell Biol. 11, 1988-1995. doi: 10.1128/MCB.11.4.1988

Yu, J., Boyapati, A., and Rundell, K. (2001). Critical Role for SV40 Small-T Antigen in Human Cell Transformation. Virology 290, 192-198. doi: 10.1006/ viro.2001.1204

Zan, J., Xu, R., Tang, X., Lu, M., Xie, S., Cang, J., et al.. (2020). RNA Helicase DDX5 Suppresses IFN-I Antiviral Innate Immune Response by Interacting With PP2A-C $\beta$ to Deactivate IRF3. Exp. Cell Res. 396 (2), 112332. doi: 10.1016/ j.yexcr.2020.112332

Conflict of Interest: The authors declare that the research was conducted in the absence of any commercial or financial relationships that could be construed as a potential conflict of interest.

Publisher's Note: All claims expressed in this article are solely those of the authors and do not necessarily represent those of their affiliated organizations, or those of the publisher, the editors and the reviewers. Any product that may be evaluated in this article, or claim that may be made by its manufacturer, is not guaranteed or endorsed by the publisher.

Copyright (C) 2021 Barski, Minnell and Maertens. This is an open-access article distributed under the terms of the Creative Commons Attribution License (CC BY). The use, distribution or reproduction in other forums is permitted, provided the original author(s) and the copyright owner(s) are credited and that the original publication in this journal is cited, in accordance with accepted academic practice. No use, distribution or reproduction is permitted which does not comply with these terms. 